\title{
Influência da escala de produção no impacto econômico da mastite em rebanhos bovinos leiteiros
}

\author{
Fabiana Alves Demeu ${ }^{1}$, Marcos Aurélio Lopes ${ }^{* 2}$, Christiane Maria Barcellos Magalhães da Rocha ${ }^{3}$, \\ Geraldo Márcio da Costa ${ }^{4}$, Glauber dos Santos ${ }^{5}$, Agnelo Franco Neto ${ }^{6}$ \\ http://dx.doi.org/10.1590/0034-737X201562020006
}

\begin{abstract}
RESUMO
Os objetivos desta pesquisa foram analisar e quantificar a influência da escala de produção no impacto econômico da mastite em rebanhos bovinos leiteiros. A pesquisa foi realizada, por meio de simulação, no sistema computacional CU\$TO MASTITE, com rebanhos leiteiros de 50, 100 e 150 vacas em lactação. Foram consideradas como de prevenção as despesas com monitoramento (cultura e antibiograma, CCST e CCSI), pré e pós dipping, vacinação, tratamento de vacas secas e manutenção de ordenhadeira. Como medidas curativas, consideraram-se os tratamentos com casos clínicos, cuja percentagem foi de 7\% das vacas em lactação. O impacto da mastite foi estimado como sendo o total de perdas, acrescido das despesas com prevenção e tratamento de casos clínicos. O aumento da escala de produção resultou em menor impacto econômico da mastite por vaca em lactação. Quando se analisou o efeito da escala de produção, os maiores responsáveis pelo impacto econômico foram, em ordem decrescente, as perdas com descarte de leite, o tratamento curativo de animais acometidos clinicamente e a redução da produção de leite. As despesas com tratamento preventivo representaram, no máximo, 11,7\% do impacto econômico, o que demonstra vantagens em investir nessa prática, que irá contribuir significativamente para diminuição do impacto econômico da mastite.
\end{abstract}

Palavras-chave: bovinocultura leiteira, economia, sanidade animal.

\section{ABSTRACT}

\section{Influence of production scale in the economic impact of mastitis in dairy cattle herds}

The objectives of this work were to analyze and to quantify the influence of the production scale on the economic impact of mastitis in dairy cattle herds. The study was conducted through a simulation in the computer system 'CU\$TO MASTITE' ('MASTITIS CO\$T'), considering dairy cattle with 50; 100 and 150 lactating cows. Monitoring expenses (culture and antibiogram, CCST and CCSI), pre and post dipping, vaccination, treatment of dry cows and milking maintenance, were considered as prevention. The treatments with clinical cases, which had a $7 \%$ percentage of lactating cows were considered as curative measures. The impact of mastitis was estimated as the total of losses plus expenses with prevention and the treatments of clinical cases. The increase of the production scale resulted in a lower economic impact of mastitis per lactating cow. When the effect of the production scale was analyzed, the losses with milk disposal, curative treatment of clinically affectedanimals and a decrease in milk production were

\footnotetext{
Submetido em 06/09/2012 e aprovado em 19/03/2015.

${ }^{1}$ Universidade Federal de Lavras, Departamento de Medicina Veterinária, Lavras, Minas Gerais, Brasil. fabianademeu@bol.com.br

${ }^{2}$ Universidade Federal de Lavras, Departamento de Medicina Veterinária, Lavras, Minas Gerais, Brasil. Bolsista do CNPq. malopes@dmv.ufla.br

${ }^{3}$ Universidade Federal de Lavras, Departamento de Medicina Veterinária, Lavras, Minas Gerais, Brasil. rochac@dmv.ufla.br

${ }^{4}$ Universidade Federal de Lavras, Departamento de Medicina Veterinária, Lavras, Minas Gerais, Brasil. gmcosta@dmv.ufla.br

${ }^{5}$ Universidade Federal de Lavras, Departamento de Medicina Veterinária, Lavras, Minas Gerais, Brasil. glauber_zoo@yahoo.com.br

${ }^{6}$ Universidade Federal de Lavras, Departamento de Medicina Veterinária, Lavras, Minas Gerais, Brasil. agnelozoo@ hotmail.com

"Autor para correspondência: malopes@dmv.ufla.br
} 
the factors that most affected the economic impact, in a descending order. Expenses with preventive treatment accounted for, at maximum, $11.7 \%$ of the economic impact, which demonstrates advantages of investing on that practice because it will contribute significantly for the reduction of the economic impact caused by mastitis.

Key words: animal health, dairy cattle, economy.

\section{INTRODUÇÃO}

Levando em consideração a grande heterogeneidade da cadeia produtiva do leite no Brasil, sua presença em todo o território nacional e o caráter dinâmico inerente ao ambiente de produção, é reconhecida a necessidade de estratificação dos produtores no que tange à comparação entre os índices alcançados pelo produtor e os obtidos em diferentes sistemas de produção. Diversos critérios de classificação dos sistemas de produção de leite têm sido adotados, cuja escolha depende dos objetivos propostos (Figueiredo \& Gomes, 2009). Dentre os mais comumente utilizados, está a escala de produção. Oliveira et al. (2001) concluíram que a escala de produção foi fator importante para alcançar índices econômicos atrativos.

Ocorre economia de escala quando os custos unitários caem com o aumento da atividade da empresa. A obtenção de uma curva em forma de "U" sugeriria a existência de ponto de escala ótimo, no qual os custos de produção são minimizados (Bressan et al., 2010). Segundo Lopes et al. (2006b), em relação à otimização da estrutura física da empresa, aumentando-se a escala de produção, até determinados níveis, o custo fixo por unidade é reduzido. Bannock et al. (2003) afirmam que o efeito da economia de escala é percebido à medida em que se aumenta a produção, mantendo-se constantes os custos fixos.

Dada a grande importância da mastite, diversos pesquisadores têm estudado seus diferentes aspectos, como a incidência de casos clínicos (Ribeiro et al., 2006; Laffranchi et al., 2001; Bueno et al., 2002); a contagem de células somáticas e a redução da produção (National Mastitis Council - NMC, 1996; Silva et al., 2002); o descarte de vacas, em virtude de mastite (Andrus et al., 1970; Burnside, 1971; Allaire, 1981; Congleton \& King, 1984; Silva et al., 2008); a prevalência, incidência e frequência de casos clínicos (Costa et al., 2001; Bueno et al., 2002; Santos \& Fonseca, 2007); a influência das células somáticas na qualidade do leite (Machado et al., 1993; Costa et al., 1999; Seegers et al., 2003; Santos \& Fonseca, 2007; Costa, 2008). No entanto, raras são as pesquisas referentes ao impacto econômico e, nas poucas encontradas na literatura (Kirk \& Bartlett, 1988;
Degraves \& Feltrow, 1993; Miller et al., 1993; Costa, 2005; Bar et al., 2008), que abordam o assunto, não há detalhamento dos indicadores técnicos e gerenciais adotados, para se poder avaliar o impacto econômico da mastite. Considerando-se os aspectos apresentados, bem como a importância do tema, realizou-se esta pesquisa, cujo objetivo foi analisar e quantificar a influência da escala de produção no impacto econômico da mastite em rebanhos bovinos leiteiros.

\section{MATERIAL E MÉTODOS}

A pesquisa foi conduzida no Laboratório de Informática Aplicada, do Departamento de Medicina Veterinária da Universidade Federal de Lavras (UFLA), por meio de simulação de dados, utilizando-se o sistema computacional “CU\$TO MASTITE”, para estimar o impacto econômico da mastite em rebanhos leiteiros, desenvolvido por Lopes et al. (2007). Os fatores utilizados (e os seus respectivos valores) foram o percentual de casos clínicos $(7 \%)$; o percentual de vacas secas (33\%); a produção diária por animal (20 kg); a frequência de ordenhas por dia (2); o intervalo de partos (15 meses); a contagem de células somáticas (500.000 células somáticas/mL); os quartos acometidos clinicamente (1); a quantidade de doses de vacina para vaca seca (3); o percentual de descarte de animais devido à mastite (4\%); o percentual de mortes em virtude da mastite/ano (1\%); o valor de aquisição da vaca $(\mathrm{R} \$ 3.000,00)$ e o valor do descarte de vacas cronicamente acometidas por mastite ( $\mathrm{R} \$ 1.314,00)$. Para definição desse valor, foi considerada a venda para abate de uma vaca de 18 arrobas a $\mathrm{R} \$ 73,00 /$ arroba. Para se calcular a influência da escala de produção sobre o impacto econômico da mastite, optou-se por rebanhos leiteiros hipotéticos, compostos por 50, 100 e 150 vacas em lactação.

Neste trabalho, a bonificação foi de $\mathrm{R} \$ 0,01 / \mathrm{kg}$ de leite para escala de $50 \mathrm{vacas} / \mathrm{dia}$, por situar-se a produção entre 500 e $1.001 \mathrm{~kg}$ de leite/dia, e $\mathrm{R} \$ 0,02 / \mathrm{kg}$ de leite comercializado, quando a produção diária ficou entre 1.001 e $3.000 \mathrm{~kg} / \mathrm{dia}$, que compreende as escalas de 100 e 150 vacas em lactação. Essa bonificação foi praticada pela Cooperativa Agrícola Alto Rio Grande, no município de Lavras, em novembro de 2008, quando o 
valor normal do litro de leite foi de $\mathrm{R} \$ 0,67$. As despesas com tratamento curativo envolveram aquelas com casos clínicos subagudos e agudos, que, neste estudo, consideraram-se com os valores de 75 e $25 \%$ respectivamente. A quantidade total de casos clínicos por mês foi obtida por meio da fórmula proposta por Santos \& Fonseca (2007):

$\%$ de mastite clínica do mês $=\frac{(\text { dicas de mastite clínica } / \text { dias do mês }) \times 100}{\text { número médio de vacas em lactação }}$

- O valor obtido foi multiplicado pela quantidade de meses do ano, estimando-se, assim, a quantidade de casos clínicos por ano.

- Custo operacional efetivo $\left(\mathrm{COE}^{2}\right)$ do tratamento curativo (caso subagudo+agudo)/vaca em lactação/ano = (valor de tratamento de casos clínicos subagudos no ano + valor de tratamento de casos clínicos agudos no ano)/quantidade de vacas em lactação, de acordo com a metodologia de Matsunaga et al. (1976).

Foram consideradas como despesas do tratamento preventivo o monitoramento (cultura e anbiograma, contagem de células somáticas no tanque e contagem de células somáticas individuais), o pré e o pósdipping, a vacinação, o tratamento de vacas secas e a manutenção de ordenhadeira (troca de mangueiras, insulfladores (troca a cada 2.500 ordenhas), óleo da bomba de vácuo e assistência técnica). Adotaram-se quatro conjuntos de ordenhadeiras para rebanhos de 50 e 100 vacas e, de seis, para rebanhos de 150 vacas. Como medidas curativas, consideraram-se apenas os tratamentos com casos clínicos, cuja percentagem foi de $7 \%$ das vacas em lactação.

O impacto da mastite (IM) foi considerado como sendo:

- $\mathrm{IM}=$ Total em perdas + tratamento preventivo + tratamento curativo, onde:

- Total de perdas $=$ morte de matrizes $(1 \% / a n o)+$ descarte prematuro de matrizes (4\% do rebanho* $\mathrm{R} \$ 1.314,00)$ + perdas em leite (redução da produção e descarte de leite durante o tratamento e período de carência do antibiótico);

- Tratamento preventivo $=$ despesas com antibiograma e cultura + CCST + CCSI + pré-dipping + pós-dipping + tratamento de vacas secas + vacinação de vacas secas + manutenção de ordenhadeira;

As perdas por redução da produção de leite decorrente da mastite, utilizadas na simulação, foram estimadas conforme a equação proposta pelo NMC(1996):

${ }^{2}$ São custos operacionais efetivos aqueles nos quais ocorre efetivamente desembolso ou dispêndio em dinheiro, tais como: mão de obra, alimentação, sanidade, reprodução, impostos (todos) e despesas diversas.
- y = - 4,7908 +0,0226x, em que "y" é a percentagem de perda de leite e "x" a contagem de células somáticas no tanque (CCST x 1.000 células/ml).

O percentual de quartos acometidos pela mastite subclínica, em função da CCST, foi estabelecido por meio da equação proposta pelo NMC (1996):

- $y=-0,2959+0,0322 x$, em que "y" é o percentual de quartos afetados pela mastite subclínica e "x" é a quantidade de células somáticas no tanque (CCST x 1000 células/mL). O valor considerado para a conversão em dólar foi US\$1.00 igual a R \$2,25.

- Tratamento curativo = despesas com aplicação do antibiótico local + antibiótico sistêmico + anti-inflamatório.

Os Valores dos insumos e produtos utilizados foram obtidos no comércio local de Lavras, em novembro de 2008.

- O valor do $\mathrm{kg}$ de leite, considerado de acordo com a produção diária, foi de $\$ 0,66, \mathrm{R} \$ 0,67$, respectivamente, para produções até $1.000 \mathrm{~kg} / \mathrm{dia}$ e acima de $1.000 \mathrm{~kg} / \mathrm{dia}$.

$\mathrm{O}$ valor do $\mathrm{kg}$ de leite, considerado para estimar as perdas, foi de $\mathrm{R} \$ 0,66$, até comercialização diária de $1.000 \mathrm{~kg} /$ leite/dia e $\mathrm{R} \$ 0,67$, quando a quantidade de leite comercializada ficou entre 1.000 e $2.000 \mathrm{~kg} / \mathrm{dia}$. Esses valores foram obtidos na Cooperativa Agrícola Alto Rio Grande, no município de Lavras, em novembro de 2008. O valor de um dólar na época da realização deste trabalho foi de $\mathrm{R} \$ 2,25$.

\section{RESULTADOS E DISCUSSÃO}

A avaliação do impacto econômico anual da mastite, por $\mathrm{kg}$ de leite, vaca em lactação e rebanho (vacas em lactação + vacas secas), em R e percentual, em função da escala de produção, pode ser observada na tabela 1. Os impactos econômicos totais da mastite, em um ano, foram de $\mathrm{R} \$ 81.910,89 ; \mathrm{R} \$ 160.481,82$ e $\mathrm{R} \$ 238.218,38$, nos rebanhos de 50, 100 e 150 vacas em lactação, respectivamente. Os valores em $\mathrm{R}$ \$ apresentam uma falsa sensação de desvantagem da maior escala de produção, pois os impactos econômicos gerados pela mastite são maiores. Isso era esperado, pois, à medida que ocorre aumento da quantidade de vacas em lactação, aumentam-se os custos variáveis (Lopes \& Carvalho, 2000), as despesas referentes ao tratamento curativo e prevenção. Esse fato pode ser verificado quando se toma por referência uma vaca em lactação, em relação à qual esses parâmetros variaram de $\mathrm{R} \$ 1.638,22$ a $\mathrm{R} \$ 1.588,12$ e de $\mathrm{R} \$ 0,2823$ a $\mathrm{R} \$ 0,2737 / \mathrm{kg}$ de leite, para rebanhos de 50 e 150 vacas em lactação, respectivamente (Tabela 1 ). A pequena diferença $(3,05 \%)$ ocorre em virtude de alguns itens envolvidos na preven- 
ção, como a postagem e a caixa térmica para envio de material para laboratório e a manutenção da ordenhadeira. Outros itens também apresentam otimização de uso de materiais, como o copo para coleta de leite, o copo para pré $e$ pós-dipping $e$ os exames de contagem de células somáticas no tanque (CCST).

Os valores relacionados com a perda por diminuição da produção apresentaram variação de $R \$ 313,61$ a $\mathrm{R} \$ 318,37$ por vaca em lactação. Esses valores foram obtidos a partir das estimativas de perdas (23.759 e $71.276 \mathrm{~kg}$ de leite/ano, respectivamente, para 50 e 150 vacas em lactação), multiplicados pelo valor do $\mathrm{kg}$ de leite ( $\mathrm{R} \$ 0,66$ e $\mathrm{R} \$ 0,68)$, o que resultou em $\mathrm{R} \$ 15.680,66$ e $R \$ 47.754,75$, para 50 e 150 vacas em lactação, respectivamente. Desses valores foram, então, calculados os percentuais das perdas. Os valores referentes às perdas por vaca em lactação/ano encontrados foram inferiores aos observados por Costa et al. (1999), ao relatarem que, no Brasil, somente as perdas com mastite subclínica podem chegar a US $\$ 317,38$. Esses pesquisadores estudaram 7.644 glândulas mamárias de 2.208 vacas leiteiras das raças holandesa, jersey, pardo suíça e mestiças, mantidas em confinamento total, parcial ou a pasto. As perdas relacionadas com a redução da produção foram estimadas pelo escore de quartos com Califórnia Matittis Test positivo, com reduções de 4,5 a $35,5 \%$.

Os custos operacionais efetivos das medidas de prevenção foram estimados em 36,12\% (US\$85,03); $29,80 \%$ (US\$63,84) e $27,24 \%$ (US\$56,32), por vaca em lactação e 11,69; 29,80 e 27,24\%/kg de leite, para as escala de produção de 50, 100 e 150 vacas, respectivamente (Tabela 1).

Os custos operacionais efetivos das medidas de prevenção por kg de leite apresentaram variações de 11,69; 8,97 e 7,96\%/kg de leite, para as escalas de 50; 100 e 150 vacas, respectivamente, enquanto as despesas com as medidas curativas foram estimadas, por $\mathrm{kg}$ de leite, para as mesmas quantidades de vacas em lactação, pois, apesar das quantidades de casos clínicos totais serem diferentes em todos os casos, foi considerado o mesmo percentual e o mesmo custo de tratamento para cada caso clínico, em todas as escalas adotadas. As diferenças nos valores por $\mathrm{kg}$ de leite foram devidas à otimização de alguns produtos utilizados na prevenção (copos de pré e de pós- dipping, manutenção de ordenhadeira, caneca de fundo preto) que, apesar de serem componentes do custo variável, influenciaram no custo operacional efetivo. Esse fato aconteceu graças ao aumento da quantidade de leite produzida, em decorrência do aumento da quantidade de vacas em lactação, que, segundo Lopes et al. (2008), é uma alternativa para aumentar a escala de produção e reduzir os custos unitários (fixo, variável e total). Outra alternativa apresentada por esses autores é aumentar a eficiência produtiva, ou seja, a produtividade por matriz, otimizando-se, assim, as despesas com mão de obra, medicamentos, inseminação artificial, impostos fixos, energia e outras e, também, por causa da maior quantidade de leite comercializada, pois, à medida que houve aumento da escala de produção, ocorreram

Tabela 1. Avaliação do impacto econômico da mastite, por kg de leite, vaca em lactação e rebanho (vacas em lactação + vacas secas), em R \$, em função da quantidade de vacas holandesas em lactação, com produção diária de 20 kg e contagem de células somáticas de 500.000 células $/ \mathrm{mL}$

\begin{tabular}{|c|c|c|c|c|c|c|}
\hline \multirow{3}{*}{ Especificação } & \multicolumn{6}{|c|}{ Quantidade de vacas em lactação } \\
\hline & \multicolumn{2}{|c|}{50} & \multicolumn{2}{|c|}{100} & \multicolumn{2}{|c|}{150} \\
\hline & $\mathbf{R} \$$ & $\%$ & $\mathbf{R} \$$ & $\%$ & $\mathbf{R} \$$ & $\%$ \\
\hline COE da prevenção/kg de leite produzido & 0,0330 & 11,69 & 0,0248 & 8,97 & 0,0218 & 7,96 \\
\hline $\mathrm{COE}$ do tratamento curativo $/ \mathrm{kg}$ de leite produzido & 0,0583 & 30,52 & 0,0583 & 30,13 & 0,0583 & 30,13 \\
\hline COE do tratamento preventivo + curativo/kg de leite produzido & 0,0913 & 42,21 & 0,0831 & 39,10 & 0,0802 & 38,09 \\
\hline Perdas de produção/kg de leite produzido & 0,1910 & 57,79 & 0,1935 & 60,90 & 0,1935 & 61,91 \\
\hline Impacto econômico/kg de leite & 0,2823 & 100,00 & 0,2766 & 100,00 & 0,2737 & 100,00 \\
\hline COE do tratamento curativo de caso subagudo/vaca afetada & 28,6800 & 20,62 & 28,6800 & 20,62 & 28,6800 & 20,62 \\
\hline COE do tratamento curativo de caso agudo/vaca afetada & 110,4300 & 79,38 & 110,4300 & 79,38 & 110,4300 & 79,38 \\
\hline COE da prevenção/vaca em lactação/ano & 191,3200 & 36,12 & 143,6300 & 29,80 & 126,7100 & 27,24 \\
\hline COE da prevenção/rebanho (vacas lactação & & & & & & \\
\hline+ vacas secas)/ano & 143,8500 & & 107,9900 & & 95,2700 & \\
\hline $\begin{array}{l}\text { COE do tratamento curativo (caso subagudo + agudo)/vaca } \\
\text { em lactação/ano }\end{array}$ & 338,3900 & 63,88 & 338,3900 & 70,20 & 338,3900 & 72,76 \\
\hline $\begin{array}{l}\text { COE do tratamento prev + curativo (caso subagudo + agudo)/ } \\
\text { vaca em lactação/ano }\end{array}$ & 529,7100 & 32,33 & 482,0200 & 30,04 & 465,1000 & 29,29 \\
\hline $\begin{array}{l}\text { Perdas de leite (redução na produção + descarte)/vaca } \\
\text { em lactação/ano }\end{array}$ & $1.108,5000$ & 67,66 & $1.122,8000$ & 69,96 & $1.123,0200$ & 70,71 \\
\hline Impacto econômico/vaca em lactação/ano & $1.638,2200$ & & $1.604,8200$ & & $1.588,1200$ & \\
\hline${ }^{1}$ Impacto econômico total (perdas + despesas)/ano & $81.910,8900$ & & $160.481,8200$ & & $238.218,3800$ & \\
\hline
\end{tabular}

Rev. Ceres, Viçosa, v. 62, n.2, p. 167-174, mar-abr, 2015 
bonificações por parte do laticínio, que foram de $\mathrm{R} \$ 0,01$ e $\mathrm{R} \$ 0,02$ para as escalas de 100 e 150 vacas, respectivamente. Isso demonstra que as medidas preventivas, que muitos julgam onerosas, trabalhosas e desnecessárias, mostram-se de excelente relação custo/benefício, corroborando os resultados obtidos por Oliveira et al. (2006), que, ao acompanharem a implantação de programas de controle de mastite, verificaram que, para cada $\mathrm{R} \$ 1,00$ de investimento, houve retorno de $\mathrm{R} \$ 2,90$ a $\mathrm{R} \$ 5,30$ de renda adicional.

Os custos de prevenção por vaca em lactação/ano, de (US\$85,03); US\$63,84 e US\$56,32, foram inferiores ao custo de tratamento curativo, que foi de US\$150,40, em todos os casos estudados. Esses valores foram obtidos a partir da divisão do valor total estimado, por ano, para os tratamentos preventivo e curativo, pela quantidade de vacas em lactação no rebanho. Pode-se verificar que o percentual do item prevenção apresentou diminuição, à medida que aumentou a escala de produção (Tabela 2). Os percentuais por vaca em lactação foram de $36,12,29,80$ e $27,24 \%$, respectivamente, para as escalas de 50, 100 e 150 vacas, enquanto os relacionados com o tratamento preventivo foram de 11,7, 8,9 e 8,0\%, respectivamente, mostrando que os maiores investimentos em prevenção resultam em menores impactos econômicos.

A prevenção é um item que deve receber grande atenção por parte dos técnicos e pecuaristas. Como pode ser observado, na tabela 2 , seu valor só não foi inferior ao das perdas com morte e desvalorização de matrizes, em todas as escalas estudadas. Esses valores evidenciam a importância de se investir e de se adotarem medidas preventivas, que incluam não somente os tratamentos preventivos, como também, medidas de manejo, prin- cipalmente na ordenha, bem como boas condições de higiene nas instalações, visando a diminuir novas infecções. Infelizmente, essa prática, de acordo com Lopes et al. (2004), não acontece. Esses autores verificaram que cuidados com a higiene do leite têm sido negligenciados por parcela significativa de produtores de leite, pois, ao fazerem o levantamento de todas as despesas operacionais efetivas, em 16 propriedades leiteiras do sul de Minas Gerais, constataram que em 50\% delas não houve nenhuma despesa com aquisição de soluções pré e pós- dipping, detergentes ácidos e alcalinos, papel toalha, desinfetantes de demais produtos utilizados na ordenha.

Se as medidas de prevenção são adotadas, tendem a aumentar os percentuais com esse item e diminuir os investimentos em tratamento, o que, consequentemente, resultará em menores perdas econômicas.

O tratamento curativo apresentou custos operacionais efetivos anuais de $\mathrm{R} \$ 16.919,63$ a $\mathrm{R} \$ 50.758,88$ (Tabela 2), representando 20,7, 21,1 e 21,3\%, respectivamente, para rebanhos com 50, 100 e 150 vacas em lactação, em relação ao impacto econômico total da mastite. Isso ocorreu, porque a maior escala considerava mais animais, e, consequentemente, uma quantidade maior de vacas acometidas por mastite clínica, o que eleva os gastos com tratamento, reflexo das despesas com tratamento de vacas clinicamente acometidas, medicamentos e seringas. Observa-se que não há uma variação expressiva, em relação ao percentual de tratamento curativo.

O custo do tratamento curativo de uma vaca com mastite, apresentando sintomas clínicos da doença, foi de $\mathrm{R} \$ 28,68$ (US\$12,75), para cada caso subagudo, e de $\mathrm{R} \$ 110,43$ (US\$49,08), para casos agudos (Tabela 1). Para

Tabela 2. Itens que compõem o impacto econômico anual da mastite em um rebanho leiteiro, em $\mathrm{R} \$$ e percentagem, em função da escala de produção, em rebanhos de 100 vacas holandesas em lactação, com produção diária de $20 \mathrm{~kg}$

\begin{tabular}{|c|c|c|c|c|c|c|}
\hline \multirow{3}{*}{ Especificação } & \multicolumn{6}{|c|}{ Quantidade de vacas em lactação } \\
\hline & \multicolumn{2}{|c|}{$\mathbf{5 0}$} & \multicolumn{2}{|c|}{100} & \multicolumn{2}{|c|}{150} \\
\hline & $\mathbf{R} \$$ & $\%$ & $\mathbf{R} \$$ & $\%$ & $\mathbf{R} \$$ & $\%$ \\
\hline \multicolumn{7}{|l|}{ Despesas } \\
\hline Tratamento preventivo & $9.566,12$ & 11,7 & $14.362,55$ & 8,9 & $19.005,76$ & 8,0 \\
\hline Tratamento curativo* & $16.919,63$ & 20,7 & $33.839,25$ & 21,1 & $50.758,88$ & 21,3 \\
\hline Despesas totais (sub total) & $26.485,75$ & 32,4 & $48.201,80$ & 30 & $69.764,64$ & 29,3 \\
\hline \multicolumn{7}{|l|}{ Perdas } \\
\hline Morte de matrizes & $1.500,00$ & 1,8 & $3.000,00$ & 1,9 & $4.500,00$ & 1,9 \\
\hline Desvalorização de matrizes & $4.518,48$ & 5,5 & $8.969,52$ & 5,6 & $13.488,00$ & 5,7 \\
\hline Descarte de leite de animais em tratamento & $33.726,00$ & 41,2 & $68.474,00$ & 42,7 & $102.711,00$ & 43,1 \\
\hline Redução na produção de leite & $15.680,66$ & 19,1 & $31.836,50$ & 19,8 & $47.754,75$ & 20,0 \\
\hline${ }^{1}$ Perdas Totais (sub total) & $55.425,14$ & 67,6 & $112.280,02$ & 70 & $168.453,75$ & $\mathbf{7 0 , 7}$ \\
\hline 2 Impacto econômico total & $81.910,89$ & 100,0 & $160.481,82$ & 100,0 & $238.218,38$ & 100,0 \\
\hline
\end{tabular}


a estimativa de casos subagudos, foram consideradas as despesas com o tratamento por meio da soma dos antibióticos locais aplicados (três dias) e a mão de obra utilizada para realizar essa atividade (10 minutos por teto acometido, com valor unitário de $\mathrm{R} \$ 0,04)$. O valor estimado para caso agudo resultou da soma dos gastos para tratamentos local e sistêmico, por um período de cinco dias. Foram considerados os valores de mão de obra, seringa e agulha para realização do tratamento sistêmico. Esses foram superiores aos $\mathrm{R} \$ 24,55$ encontrados por Costa et al. (2005), que não mencionaram se os valores encontrados foram para casos subagudos ou agudos. $\mathrm{O}$ custo do tratamento curativo de uma vaca em lactação/ ano foi estimado em $\mathrm{R} \$ 338,39$ (US\$150,40), para todos os casos estudados.

$\mathrm{O}$ item que provocou o maior impacto econômico foi o das despesas com descarte de leite de animais em tratamento de mastite clínica (Tabela 2). Os percentuais variaram de 41,2 a $43,1 \%$, respectivamente, para as escalas de 50 e 150 vacas em lactação. A variação do valor ocorreu em função do aumento da quantidade de casos clínicos, em virtude da maior quantidade de animais em lactação, e não da frequência média anual de mastite clínica.

A questão relacionada com o descarte do leite, item de grande importância nas perdas econômicas e no impacto econômico da mastite, ainda se torna um agravante maior, quando se analisa a questão do destino desse leite e da poluição ambiental. Pesquisadores têm recomendado estudos adicionais com relação à utilização desse leite, para o aleitamento artificial de bezerros, o que era uma prática recomendada há alguns anos. Batista et al. (2008) verificaram que o aleitamento de bezerras, com o leite de vacas em tratamento com mastite, pode resultar em bactérias patogênicas mais resistentes e na diminuição da flora protetora, levando à diminuição das respostas imunológicas e, talvez, à maior quantidade de casos de criptosporidiose. Com relação à questão ambiental, se esse leite não tem destino adequado, haverá a necessidade de se pensar em um descarte apropriado, após tratamento de efluentes, como previsto na resolução CONAMA n³57/2005, no capítulo IV, artigo 24 , que estabelece que "os efluentes de qualquer fonte poluidora somente poderão ser lançados, direta ou indiretamente, nos corpos de água, após o devido tratamento e desde que obedeçam às condições, padrões e exigências dispostos" (Brasil, 2005), uma vez que não há uma utilização racional recomendada. A desvalorização de matrizes acometidas por mastite crônicas (valor obtido pela diferença do valor de aquisição de animais produtivos e venda do animal improdutivo para o abate), por causa do descarte involuntário (descarte de animais acometidos por mastite), representou de 5,5 a $5,7 \%$ do im- pacto total, de acordo com as escalas de produção estudadas (Tabela 2). A desvalorização dos animais é um item que onera o produtor, uma vez que, para cada animal descartado serão necessários investimentos para reposição. Lopes et al. (2009b) verificaram que a desvalorização das vacas descartadas involuntariamente é bastante acentuada, uma vez que as fêmeas que não estavam nesse grupo renderam uma receita de $\mathrm{R} \$ 2.680,00$; enquanto as fêmeas comercializadas por descarte involuntário, das quais os pesquisadores citam problemas mamários, auferiram uma receita de somente $\mathrm{R} \$ 882,00 /$ vaca, o que representa 67,09\% de desvalorização. Valores tão baixos se devem ao fato de que esses animais são comercializados pelo valor pago por arroba da vaca gorda, enquanto as vacas oriundas de descarte voluntário são animais eliminados de um rebanho e vendidos para outro, pois ainda possuem capacidade produtiva.

A receita bruta foi de $\mathrm{R} \$ 195.014,86, \mathrm{R} \$ 395.779,98$ e $\mathrm{R} \$ 593.696,25$, o que correspondeu à soma dos valores apurados com a venda de leite $(98,19,98,23$ e $98,23 \%)$ e animais $(1,81,1,77, \mathrm{e} 1,77 \%)$, para escalas de 50,100 e 150 vacas, respectivamente.

Quanto à venda de animais, não há menção na literatura de qual percentual seria o ideal para esses valores. No entanto, essas estimativas são pequenas, quando comparadas aos valores obtidos por Lopes et al. (2008), que encontraram 7,55, 16,26 e 3,14\%, e Lopes et al. (2006a), de 20,29, 7,29 e 9,70\%, para pequenos, médios e grandes produtores. A diferença dos percentuais é devida ao fato de que, neste estudo, foi considerado somente o descarte involuntário por mastite, enquanto nos outros estudos mencionados foram consideradas todas as vendas de animais, o que possibilita maior receita. Lopes et al. (2009a) encontraram de 15 a $20 \%$ da receita bruta proveniente da venda de animais, o que também está bem acima dos resultados observados neste estudo.

Os percentuais dos custos de prevenção, em relação à renda bruta, foram estimados em: 4,91, 3,63 e 3,20\%, enquanto os referentes ao tratamento curativo foram de $8,68,8,55$ e 8,55\%, para escalas de produção de 50, 100 e 150 vacas respectivamente.

As perdas totais apresentaram o maior impacto percentual, em relação à renda bruta do sistema de produção, sendo de 28,42, 28,37 e 28,37\%, para escalas de 50, 100 e 150 vacas, e foram compostas por: morte de matrizes $(0,77,0,76$ e $0,76 \%)$, desvalorização de matrizes $(2,32,2,27$ e 2,27\%), descarte de leite de animais em tratamento $(17,29,17,30$ e 17,30\%), redução na produção $(8,04,8,04$ e 8,04\%), para escala de produção de 50,100 e 150 vacas, respectivamente. O impacto econômico total da mastite resultou em 42,00, 40,55 e $40,12 \%$, para as mesmas escalas estudadas. 
O descarte de leite de animais em tratamento teve a maior representatividade entre as perdas analisadas, sendo responsável por até $17,30 \%$ do impacto econômico causado pela mastite.

As perdas devidas à morte de matrizes apresentaram aumento de percentual, em relação à renda bruta dos sistemas de produção, por causa da quantidade de animais que morreram, nas escalas de 50 e 100 vacas, que foi de meia e de uma vaca, respectivamente. Dentre as perdas, o descarte de leite de animais em tratamento foi o responsável pelo maior percentual do impacto em todas as escalas estudadas. Isso pode ser atribuído ao percentual de casos clínicos adotado (7\%), que está bem acima de $1 \%$, que é um indicativo de saúde da glândula mamária (Santos \& Fonseca, 2007). No entanto, vários pesquisadores têm relatado valores bem maiores, chegando a 63,7\% (Degraves \& Fetrow, 1993).

O impacto econômico gerado é causado pelas despesas, perdas e penalização de pagamento. As perdas podem ser classificadas como visíveis e aquelas não perceptíveis para o produtor. Entre as visíveis se encaixam o descarte de leite de animais em tratamento. A redução da produção de leite é uma causa de impacto não perceptível, uma vez que a diminuição da produção ocasionada por mastite subclínica muitas vezes passa despercebida pelo pecuarista, o que reflete em menor produção e, na maioria das vezes, o produtor nem tem ideia de sua ocorrência.

\section{CONCLUSÕES}

$\mathrm{O}$ aumento da escala de produção resultou em menor impacto econômico da mastite por vaca em lactação. O maior impacto econômico nas menores escalas evidencia a necessidade de otimização do uso dos materiais e equipamentos utilizados na prevenção. Quando se analisou o efeito da escala de produção, os maiores responsáveis pelo impacto econômico foram, em ordem decrescente, as perdas com descarte de leite, o custo do tratamento curativo de animais acometidos clinicamente e a redução da produção de leite. O elevado impacto evidencia a necessidade de monitoramento das mastites clínica e subclínica e de adoção de medidas preventivas, para se diminuírem os prejuízos ocasionados pela doença. As despesas com tratamento preventivo representaram, no máximo, $11,7 \%$ do impacto econômico, o que mostra as vantagens de se investir nessa prática, pois isso irá contribuir significativamente para a diminuição do impacto econômico da mastite.

\section{REFERÊNCIAS}

Allaire FR (1981) Economic consequences of replacing cows with genetically improved heifers. Journal of Dairy Science, 64:1985-1995.
Andrus DF, Freeman AE \& Eastwood BR (1970) Age distribution and herd life expectancy in iowa dairy herds. Journal of Dairy Science, 53:764-771.

Bannock GRE, Baxter RE \& Davis E (2003) The Penguin Dictionary of Economics. $7^{\circ}$ ed. London, Penguin Books. 416 p.

Bar D, Tauer LW, Bennett G, Gonzalez RN, Hertl JA, Schukken YH, Schulte HF, Welcome FL \& Gröhn YT (2008) The cost of generic clinical mastitis in dairy cows as estimated by using dynamic programming. Journal of Dairy Science, 91:2205-2214.

Batista CG, Coelho SG, Rabelo E, Lana AMQ, Carvalho AU, Reis RB \& Saturnino HM (2008) Desempenho e saúde de bezerras alimentadas com leite sem resíduo de drogas antimicrobianas ou leite de vacas tratadas contra mastite, adicionado ou não de probiótico. Arquivo Brasileiro de Medicina Veterinária e Zootecnia, 60:185-191.

Brasil (2005) Ministério do Meio Ambiente. Conselho Nacional do Meio Ambiente. Resolução n ${ }^{\circ} 357$. Dispõe sobre a classificação dos corpos de água e diretrizes ambientais para o seu enquadramento, bem como estabelece as condições e padrões de lançamento de efluentes. Disponível em: <http://portal.saude.gov.br/portal/arquivos/pdf/ resolucao_conama_357.pdf>.Acessado em: 5 de março de 2009.

Bressan VGF, Braga MJ \& Bressan AA (2010) Eficiência e economia de escala em cooperativas de crédito: uma abordagem de fronteira estocástica de custo com dados em painel. Advances in Scientific and Applied Accounting, 3:335-352.

Bueno VFF, Nicolau ES, Mesquita AJ, Ribeiro AR, Silva JAB, Costa EO, Coelho KO \& Neves RBS (2002) Mastite bovina clínica e subclínica, na região de Pirassununga, SP: frequências e redução na produção. Ciência Animal Brasileira, 3:47-52.

Burnside EB (1971) Cow-indexing for potential procedure and culling levels dams of dairy bulls. Journal of Dairy Science, 53:195-199.

Congleton WR \& King LW (1984) Profitability of dairy cow herd life. Journal of Dairy Science, 67:661-674.

Costa EO, Garino Júnior F \& Watanabe ET (2001) Proporção de ocorrência da mastite clínica em relação à subclínica correlacionada com os principais agentes etiológicos. Revista do Napgama, 4:10-13.

Costa EO, Mota R \& Santos FGB (2005) Contagem de células somáticas de amostras de leite de glândulas mamárias de fêmeas bovinas em lactação infectadas por microrganismos dos gêneros Streptococcus, Staphylococcus e Corynebacterium. Revista Napgama, 8:03-07.

Costa EO, Ribeiro AR \& Watanabe ET (1999) Mastite sub-clínica: prejuízos causados e os custos de prevenção em propriedades leiteiras. Revista do Napgama, 2:16-20.

Costa GM (2008) Mastite bovina em rebanhos leiteiros da região sul do estado de Minas Gerais. Tese de Doutorado. Universidade Federal de Minas Gerais, Belo Horizonte. 123p.

Degraves FJ \& Fetrow J (1993) Economics of mastitis and mastitis control. Veterinary Clinics of North America: Food Animal Practice, 9:421-434.

Figueiredo AM \& Gomes AL (2009) Considerações sobre estratificação de produtores de leite em Minas Gerais. In: XLVII Congresso da Sociedade Brasileira de Economia, Administração e Sociologia Rural, Porto Alegre. Anais, SOBER. 3p.

Kirk JH \& Bartlett PC (1988) Economic impact of mastits in Michigan Hostein dairy herss using a computerized records system. AgriPratice, 9:03-06.

Laffranchi A, Müller EE, Freitas JC, Pretto-Giordano LG, Dias JA \& Salvador R (2001) Etiologia das infecções ultra-mamárias em vacas primíparas ao longo dos primeiros quatro meses de lactação. Ciência Rural, 32:1027-1032.

Lopes MA, Cardoso MG \& Demeu FA (2009a) Influência de diferentes índices zootécnicos na composição e evolução de rebanhos bovinos leiteiros. Ciência Animal Brasileira, 10:446-453. 
Lopes MA, Demeu FA, Santos G \& Cardoso MG (2009b) Impacto econômico do intervalo de partos em rebanhos bovinos leiteiros. Ciência e Agrotecnologia, 33:1908-1914.

Lopes MA, Dias AS, Carvalho FM, Lima ALR, Cardoso MG \& Carmo EA (2008) Efeito da escala de produção nos resultados econômicos de sistemas de produção de leite na região de Lavras (MG, Brasil), em 2004 e 2005. Archivos Latinoamericanos de Produção Animal, 16:121129.

Lopes MA, Lima ALR, Carvalho F de M, Reis RP, Santos IC \& Saraiva FH (2004) Efeito do tipo de sistema de criação nos resultados econômicos de sistemas de produção de leite na região de Lavras (MG). Ciência e Agrotecnologia, 28:1177-1189.

Lopes MA, Lima ALR, Carvalho F de M, Reis RP, Santos IC \& Saraiva FH (2006b) Efeito da escala de produção nos resultados econômicos de sistemas de produção de leite na região de Lavras (MG): um estudo multicasos. Boletim de Indústria Animal, 63:177-188

Lopes MA, Santos G, Costa GM \& Lopes NM (2007) Desenvolvimento de um sistema computacional para avaliar o impacto econômico da mastite em rebanhos leiteiros. In: Congresso Brasileiro de Agroinformática, São Pedro. Anais, SBIAgro. p.1-5.

Lopes MA, Lima ALR, Carvalho FM, Reis RP, Santos IC \& Saraiva FH (2006a) Efeito da escala de produção nos resultados econômicos de sistemas de produção de leite na região de Lavras (MG): um estudo multicasos. Boletim da Indústria Animal, 63:177-188.

Lopes MA \& Carvalho FM (2000) Custo de produção de leite. Lavras, UFLA. 42p. (Boletim agropecuário, 33).

Machado SG, Cardoso VL, Silva AMS \& Freitas MAR (1993) Influência da ocorrência da mastite na produção de leite e duração da lactação. In: 30a Reunião Anual da Sociedade Brasileira de Zootecnia, Rio de Janeiro. Anais, SBZ. p.587.
Matsunaga M, Bemelmans PF, Toledo PEN de, Dulley RD, Okawa H \& Pedroso IA (1976) Metodologia do custo de produção utilizada pelo IEA. Agricultura em São Paulo, 23:123-139.

Miller RH, Paape MJ \& Fulton LA (1993) The relationship of milk somatic cell count to milk yields for holstein heifers after first calving. Journal of Dairy Science, 76:728-733.

NMC - National Mastitis Council (1996) Current concepts of bovine mastitis. $4^{\mathrm{a}}$ ed. Washington, Madison. 64p.

Oliveira VM, Carneiro AV \& Silva MR (2006) Benefícios de um programa de controle de mastite bovina em condições brasileiras de criação. In: $6^{\circ}$ Congresso Panamericano de Leite, Juiz de Fora. Anais, UFJF. CD-ROM.

Oliveira TBA, Figueredo RS, Oliveira MW \& Nascif C (2001) Índices técnicos e rentabilidade na pecuária leiteira. Scientia Agrícola, 58:687692.

Ribeiro MER, Petrini LA, Barbosa RS, Zanela MB, Gomes JF, Stumpf Junior W \& Schramm R (2006) Ocorrência de mastite causada por nocardia spp. em rebanhos de unidades de produção leiteira no sul do rio grande do sul. Revista Brasileira de Agrociência, 12:471-473.

Santos MV \& Fonseca LFL (2007) Estratégias para controle de mastite e melhoria da qualidade do leite. São Paulo, Manole. 313p.

Seegers H, Fourichon C \& Beaudeau F (2003) Production effects related to mastitis and mastitis economics in dairy cattle herds. Veterinary Research, 34:475-491.

Silva FC, Bergamasco AF \& Vendite LL (2002) Modelos de simulação para análise e apoio à decisão em agrossistemas. Revista Biociências, 8:07-17.

Silva LAF, Coelho KO, Machado PF, Silva MAM, Moura MI, Barbosa VT, Goulart MM \& Goulart DS (2008) Causas de descarte de vacas da raça holandesa confinadas em uma população de 2.083 bovinos (2000-2003). Ciência Animal Brasileira, 9:383-389. 